\title{
Caracterización del cáncer gástrico abordado por laparoscopia en un centro del caribe colombiano
}

\author{
Characterization of gastric cancer approached by laparoscopy in a \\ center in the Colombian Caribbean
}

\author{
Juan Carlos Hoyos-Valdelamar $\mathbb{D}^{\mathbb{D}}$, Jaime Andrés Hernández-Valdelamar² $\mathbb{D}$, \\ Ana María Santos-Arrieta ${ }^{2}$ (D)
} Médico, especialista en Cirugía gastrointestinal y endoscopia digestiva, Hospital Universitario del Caribe, Cartagena, Colombia.
Médico, residente de Cirugía General, Universidad de Cartagena, Cartagena, Colombia.

\section{Resumen}

Introducción. El cáncer gástrico es una enfermedad con gran carga de salud a nivel mundial, con altas cifras de incidencia y prevalencia, por lo cual requiere de tratamiento seguros que permitan una baja morbilidad, una mejor sobrevida y calidad de vida. Dentro de los tratamientos disponibles actualmente, la gastrectomía laparoscópica se plantea como un procedimiento menos mórbido, seguro y con buenos resultados oncológicos.

Métodos. Estudio observacional descriptivo que incluyó pacientes con cáncer gástrico sometidos a gastrectomía por vía laparoscópica en el Hospital Universitario del Caribe durante un periodo de 5 años.

Resultados. En la población total el promedio de la edad fue del 68,3 \pm II,4 años, el índice de masa corporal de $23,6 \pm 2,6 \mathrm{~kg} / \mathrm{m}^{2}$ y la estancia hospitalaria de $\mathrm{I} 8,7 \pm \mathrm{II}, 7$ días. El $60 \%$ de los pacientes eran hombres y el $55 \%$ estaban afiliados al régimen contributivo, provenientes del servicio de urgencias en $77,5 \%$, con hipertensión arterial en $62,5 \%$, diabetes mellitus en $40,0 \%$ y otras alteraciones cardiovasculares en un $7,5 \%$. En cuanto al acto quirúrgico, $35 \%$ ingresaron con riesgo prequirúrgico bajo y 7 pacientes (17,5\%) requirieron transfusión prequirúrgica. Se realizó gastrectomía total en la mitad y subtotal en la otra mitad de los pacientes, con anestesia general. La lesión macroscópica se halló en un $30 \%$ en el cuerpo gástrico, $30 \%$ en el fondo y $30 \%$ en el antro, siendo adenocarcinoma en un $87,5 \%$. Los tipos más comunes según la clasificación de Bormann fueron el IV en el $55 \%$ y el III en el $27,5 \%$, con estadio IIIB en un $37,5 \%$ y IIB en un $40 \%$.

Discusión. La gastrectomía laparoscopia en el Hospital Universitario del Caribe, en Cartagena, Colombia, presenta buen perfil de seguridad, con igual tendencia que el perfil epidemiológico reportado a nivel mundial.

Palabras Clave: neoplasias gástricas; adenocarcinoma; gastrectomía; laparoscopía; resultado del tratamiento.

Fecha de recibido: 31/06/2019 - Fecha de aceptación: 21/08/2019

Correspondencia: Juan Carlos Hoyos Valdelamar, MD. Dirección postal: 130008. Teléfono: 3135805228.

juancarloshoyosval@hotmail.com

Citar como: Hoyos-Valdelamar JC, Hernández-Valdelamar JA, Santos-Arrieta AM. Caracterización del cáncer gástrico abordado por laparoscopia en un centro del caribe colombiano. Rev Colomb Cir. 2020;35:575-82. https://doi.org/10.30944/20117582.795

Este es un artículo de acceso abierto bajo una Licencia Creative Commons - BY-NC-ND https://creativecommons.org/licenses/by-ncnd/4.0/deed.es 


\begin{abstract}
Introduction. Gastric cancer is a disease with a great health burden worldwide, with high incidence and prevalence figures, for which it requires safe treatment that allows low morbidity, better survival and quality of life. Among the currently available treatments, laparoscopic gastrectomy is considered a less morbid and safe procedure with good oncological results.

Methods. Descriptive observational study that included patients with gastric cancer who underwent laparoscopic gastrectomy at the Hospital Universitario del Caribe during a period of 5 years.

Results. In the total population, the age was $68.3 \pm$ II.4 years, the body mass index was $23.6 \pm 2.6 \mathrm{~kg} / \mathrm{m}^{2}$, and the hospital stay was $18.7 \pm$ II.7 days. A $60 \%$ of the patients were men and $55 \%$ of the contributory regimen, coming from the emergency department in $77.5 \%$, with arterial hypertension in $62.5 \%$, diabetes mellitus in $40 \%$ and other cardiovascular alterations in $7.5 \%$. Regarding the surgical act, $35 \%$ were admitted with low pre-surgical risk and seven patients (17.5\%) required pre-surgical transfusion. Total gastrectomy was performed in half and subtotal in the other half of the patients, under general anesthesia. The macroscopic lesion was found in $30 \%$ in the gastric body, $30 \%$ in the fundus, and $30 \%$ in the antrum, with $87.5 \%$ being adenocarcinoma. The most common types according to the Bormann classification were IV in $55 \%$ and III in $27.5 \%$, with stage IIIB in $37.5 \%$ and IIB in $40 \%$.
\end{abstract}

Conclusion. Laparoscopic gastrectomy in the Hospital Universitario del Caribe, Cartagena, Colombia, presents a good safety profile with the same tendency of epidemiological profile to the one reported worldwide.

Keywords: stomach neoplasms; adenocarcinoma; gastrectomy; laparoscopy; treatment outcome.

\section{Introducción}

El cáncer gástrico es un problema de salud pública con gran impacto a pesar de la disminución en su incidencia, que alcanza 95I.00o casos por año a nivel mundial ${ }^{\mathrm{I}}$. Sigue siendo la tercera causa de muerte relacionada con cáncer en todo el mundo, después del cáncer de pulmón e hígado, con un total de 723.000 muertes anuales ${ }^{\text {. }}$

La distribución geográfica del cáncer gástrico está caracterizada por una amplia variación, registrando la más alta incidencia en regiones como Asia Oriental (Japón y China), Europa Oriental y Centroamérica, mientras que una baja incidencia se registra en Asia del Sur, el Norte y Este de África, Norte América, Australia y Nueva Zelanda ${ }^{ }$. Actualmente tres países concentran el $60 \%$ de los casos de cáncer gástrico en el mundo: Japón, China y Corea. En Latinoamérica, la incidencia y la mortalidad por cáncer gástrico disminuyeron uniformemente, tanto en hombres como en mujeres ${ }^{2}$, mientras en Sur América, Globocan reportó 47.244 casos de cáncer gástrico (29.312 hombres y I7.932 mujeres), lo que representó el I2 \% de todos los cánceres reportados ${ }^{2}$.
En Colombia, en el 2015 el cáncer gástrico fue la primera causa de muerte en ambos sexos, con el I4,I \% de las defunciones entre todos los tipos de cáncer ${ }^{3}$. El estudio REGATE mostró como características de la población colombiana afectada por cáncer gástrico un promedio de edad de 62 años, con predominio del sexo masculino (65\% de los pacientes), presencia de síntomas gástricos en el $98 \%$ de los casos, y diagnóstico en promedio 6 meses después de iniciados los síntomas ${ }^{4}$, observando además patrones claramente definidos con alta incidencia, prevalencia y mortalidad en la zona Andina.

En el departamento de Bolívar, en Colombia, entre 2007 y 201 se calculó una incidencia de 5,4X Ioo.00o hombres y de 3,4 x I00.000 mujeres, con una tasa de mortalidad de 4,3 defunciones por IO0.000 hombres y 2,5 defunciones por IO0.000 mujeres ${ }^{5}$, mientras en la ciudad de Cartagena de Indias, capital del Departamento, en el año 2013 se presentaron 20 defunciones por cáncer gástrico, representando el $0,5 \%$ de muertes por cáncer, con una tasa de mortalidad de 2,I muertes por I00.00o habitantes ${ }^{6}$. 
En el Hospital Universitario del Caribe, desde 2007 a 2009 se trataron 6I pacientes con cáncer gástrico, de los cuales 56 casos (91,8\%) fueron adenocarcinomas, el 57,3\% eran mayores de 60 años, con una relación hombre : mujer de 3:I; la localización más frecuente fue en antro $(44,3 \%)$ y se realizó gastrectomía con fines paliativos en un $33,3 \%$, gastrectomía curativa relativa en el $50 \%$ y gastrectomía curativa absoluta en el I6,6 \% 7 , sin reportar mortalidad o complicaciones durante el proceso de tratamiento.

Actualmente, el cáncer gástrico representa un desafío clínico importante porque la mayoría de los casos se diagnostican en una etapa avanzada, con un pronóstico de sobrevida bajo y opciones de tratamiento limitadas. El tratamiento del cáncer gástrico, en caso de ser resecable, tiene como pilar la gastrectomía, cuya técnica abierta es la referencia con la que se comparan todas las nuevas tecnologías, incluida la cirugía laparoscópica, de amplia aceptación en el ámbito académico ${ }^{8}$, por lo que se han ampliado sus indicaciones, con buen consenso para indicarla en casos de cáncer gástrico sin evidencia de ganglios linfáticos metastásicos 9 .

El objetivo de este estudio fue evaluar las características sociodemográficas, clínicas e histológicas de los pacientes diagnosticados con cáncer gástrico que fueron sometidos a gastrectomía laparoscópica en el Hospital Universitario del Caribe, durante el periodo de 5 años comprendido entre junio de 2013 a junio de 2018.

\section{Métodos}

Estudio observacional descriptivo que tomó como población sujeta de estudio los pacientes con diagnóstico de cáncer gástrico que fueron atendidos en el Hospital Universitario del Caribe por el servicio de cirugía laparoscópica entre 20I3 y 20I8. Los criterios de inclusión fueron ser mayores de 18 años, con diagnóstico de cáncer gástrico, sometidos a gastrectomía laparoscópica. Se excluyeron los pacientes en estado de gravidez o con enfermedad autoinmune concomitante.

La recolección de la información de las historias clínicas se realizó de forma retrospectiva, luego de identificar el grupo de pacientes que cumplieron con los criterios de inclusión, haciendo una revisión de la base de datos institucional, donde se encuentran consignados los procedimientos laparoscópicos. En todos los pacientes seleccionados se indagaron variables sociodemográficas como edad y sexo, régimen de seguridad social en salud, datos clínicos como el estado nutricional, procedencia (remitido o no), servicio de origen, antecedentes personales patológicos de hipertensión arterial, diabetes mellitus, paraclínicos como albúmina y hemoglobina pre quirúrgica y pos quirúrgica; dentro de los datos intraoperatorios se identificó la clasificación ASA, tipo de anestesia, tipo de gastrectomía, localización anatómica de la lesión, clasificación de Borrmann, tipo histológico, estadio, hallazgo de pérdidas sanguíneas, complicaciones, transfusiones, estancia hospitalaria y mortalidad.

Se hizo análisis descriptivo de los datos cualitativos, reportando frecuencias absolutas y relativas; las variables cuantitativas se expresaron con medidas de tendencia central y de dispersión, según criterio de normalidad por la prueba Shapiro-Wilk. La comparación de variables cualitativas se realizó con $\mathrm{Chi}^{2}$ o test exacto de Fisher según fuera necesario, mientras las variables cuantitativas se compararon con la prueba t Student. Un valor de $\mathrm{p}<0,05$ fue considerado como estadísticamente significativo.

\section{Resultados}

En el periodo de estudio se identificaron 40 historias clínicas de pacientes que cumplieron los criterios de selección. El promedio de edad fue de $68,3 \pm$ II,4 años, y el $60 \%$ eran hombres (tabla I). El promedio del índice de masa corporal (IMC) fue de 23,6 $\pm 2,6 \mathrm{~kg} / \mathrm{m}^{2}$, observándose en un $60 \%$ peso normal, seguido de sobrepeso en el $35 \%$. Un $55,5 \%$ de los pacientes estaban afiliados al régimen contributivo de salud, 27,5\% de los pacientes fueron remitidos y $77,5 \%$ procedían del servicio de urgencias. Los antecedentes personales más frecuentes fueron hipertensión arterial $(62,5 \%)$ y diabetes mellitus (40 \%). Los paraclínicos mostraron valores promedios de 
Tabla 1. Características sociodemográficas, antecedentes personales y exámenes de laboratorio en los pacientes estudiados $(n=40)$.

\begin{tabular}{|c|c|c|}
\hline Variable & $\mathbf{n}$ & $\%$ \\
\hline Edad, en años (Promedio y DE) & $68,3 \pm 11,4$ & \\
\hline \multicolumn{3}{|l|}{ Sexo } \\
\hline Femenino & 16 & 40,0 \\
\hline Masculino & 24 & 60,0 \\
\hline IMC, en kg/m2 (Promedio y DE) & $23,6 \pm 2,6$ & \\
\hline Bajo peso & 2 & 5,0 \\
\hline Peso normal & 24 & 60,0 \\
\hline Sobrepeso & 14 & 35,0 \\
\hline Obesidad & 0 & 0,0 \\
\hline \multicolumn{3}{|l|}{ Régimen de Seguridad Social en Salud } \\
\hline Contributivo & 22 & 55,0 \\
\hline Subsidiado & 18 & 45,0 \\
\hline \multicolumn{3}{|l|}{ Origen del paciente } \\
\hline No remitido & 29 & 72,5 \\
\hline Remitido & 11 & 27,5 \\
\hline \multicolumn{3}{|l|}{ Servicio de ingreso al hospital } \\
\hline Urgencia & 31 & 77,5 \\
\hline Consulta externa & 9 & 22,5 \\
\hline \multicolumn{3}{|l|}{ Antecedentes personales } \\
\hline Hipertensión arterial & 25 & 62,5 \\
\hline Diabetes mellitus & 16 & 40,0 \\
\hline Cardiovascular & 3 & 7,5 \\
\hline Asma & 1 & 2,5 \\
\hline $\begin{array}{l}\text { Enfermedad pulmonar obstructiva } \\
\text { crónica (EPOC) }\end{array}$ & 1 & 2,5 \\
\hline \multicolumn{3}{|l|}{ Hemoglobina $(n=33)$ (Promedio y DE) } \\
\hline Prequirúrgica (g/L) & $9,3 \pm 1,9$ & \\
\hline Posquirúrgica (g/L) & $10,1 \pm 1,3$ & \\
\hline \multicolumn{3}{|l|}{ Albúmina prequirúrgica $(\mathrm{g} / \mathrm{dL})$} \\
\hline$<2$ & 0 & 0,0 \\
\hline $2-2,5$ & 1 & 2,5 \\
\hline $2,6-3,0$ & 14 & 35,0 \\
\hline $3,1-3,5$ & 14 & 35,0 \\
\hline$>3,5$ & 2 & 5,0 \\
\hline No reportado & 9 & 22,5 \\
\hline
\end{tabular}

*DE: desviación estándar; IMC: índice de masa corporal

hemoglobina prequirúrgica y posquirúrgica de $9,3 \%$ y IO,I \% respectivamente; por su parte la albúmina prequirúrgica osciló entre 2,6 y 3,6 g/dl en un $79 \%$ de los pacientes.

La lesión se localizó en fondo, cuerpo y antro gástrico, con la misma proporción de 30\% cada uno. La clasificación de Borrmann más frecuente fue IV en el $55 \%$ de los casos, seguido de III en el $27,5 \%$, y el tipo histológico más común fue el adenocarcinoma, en el $87,5 \%$. Los estadios más frecuentes fueron el IIB y IIIB, con $40 \%$ y $37,5 \%$, respectivamente.

Se encontró una clasificación de ASA (American Society of Anesthesiologists) I en el $35 \%$, seguido de ASA II y III con $25 \%$ cada uno. La anestesia fue general en $97,5 \%$ y en la mitad de los pacientes se realizó gastrectomía total. La pérdida sanguínea se observó en el $5 \%$, y se transfundieron el $55 \%$ de los pacientes, siendo posquirúrgica en un $37,5 \%$. En $2,5 \%$ se presentaron complicaciones. El promedio de estancia hospitalaria fue de $\mathrm{I} 8$ días $(\mathrm{DE}=\mathrm{II}, 7)$ y no se observaron casos fatales en los 30 días posquirúrgicos (tabla 2).

Al comparar las características sociodemográficas entre los sexos, no se observaron diferencias estadísticamente significativas. En los paraclínicos, se observó una mayor frecuencia de albúmina baja, entre 2 y $2,5 \mathrm{~g} / \mathrm{dL}$, en las mujeres $(62,7 \%)$, comparado con los hombres $(4,2 \%)$, p<0,ooo (tabla 3). Este mismo análisis, comparando los aspectos de la evolución pre, intra y posquirúrgica, entre hombres y mujeres no mostró diferencias significativas (tabla 4).

\section{Discusión}

En cuanto al perfil epidemiológico, en este estudio se encontró lo mismo que en la literatura mundial, donde es más frecuente en personas de sexo masculino, con edad promedio de 68 años, de peso normal, afiliados al régimen contributivo de seguridad social, que ingresaron al hospital consultando por el servicio de urgencias. La lesión correspondió a adenocarcinomas, de localización proximal, con estadio IIB o IIIB, y una clasificación del tumor $\mathrm{T}_{4}$ (tumor que compromete estructuras adyacentes), es decir, avanzado.

En 2008, Adrada y colaboradores " ${ }^{\text {II }}$ documentaron que, en su población en Cauca, Colombia, el cáncer gástrico tiene el mismo comportamiento epidemiológico que clásicamente se ha encontrado en otras investigaciones, como que es más frecuente en hombres, aumenta con la edad, predomina el adenocarcinoma, de localización proximal y en estadios avanzados. 
Tabla 2. Anestesia, cirugía y clasificación de la lesión en los pacientes operados.

\begin{tabular}{|c|c|c|}
\hline Variable & $\mathbf{n}$ & $\%$ \\
\hline \multicolumn{3}{|l|}{ Clasificación ASA } \\
\hline 1 & 14 & 35,0 \\
\hline II & 10 & 25,0 \\
\hline III & 10 & 25,0 \\
\hline IV & 6 & 15,0 \\
\hline \multicolumn{3}{|l|}{ Tipo de anestesia } \\
\hline General & 39 & 97,5 \\
\hline Mixta & 1 & 2,5 \\
\hline \multicolumn{3}{|l|}{ Cirugía realizada por laparoscopia } \\
\hline Gastrectomía subtotal & 20 & 50,0 \\
\hline Gastrectomía total & 20 & 50,0 \\
\hline \multicolumn{3}{|l|}{ Localización anatómica de la lesión } \\
\hline Unión esofagogástrica & 1 & 2,5 \\
\hline Fondo gástrico & 12 & 30,0 \\
\hline Cuerpo gástrico & 12 & 30,0 \\
\hline Antro gástrico & 12 & 30,0 \\
\hline No reportado & 3 & 7,5 \\
\hline \multicolumn{3}{|l|}{ Clasificación de Borrmann } \\
\hline Tipo 1 & 1 & 2,5 \\
\hline Tipo 2 & 2 & 5,0 \\
\hline Tipo 3 & 11 & 27,5 \\
\hline Tipo 4 & 22 & 55,0 \\
\hline No reportado & 4 & 10,0 \\
\hline \multicolumn{3}{|l|}{ Tipo histológico } \\
\hline Adenocarcinoma & 35 & 87,5 \\
\hline Sin datos & 5 & 12,5 \\
\hline \multicolumn{3}{|l|}{ Estadio } \\
\hline IIB & 16 & 40,0 \\
\hline IIC & 1 & 2,5 \\
\hline IIIA & 3 & 7,5 \\
\hline IIIB & 15 & 37,5 \\
\hline No reportado & 5 & 12,5 \\
\hline Pérdida sanguínea & 2 & 5,0 \\
\hline Complicaciones & 1 & 2,5 \\
\hline Transfusiones & 22 & 55,0 \\
\hline Prequirúrgica & 7 & 17,5 \\
\hline Posquirúrgica & 15 & 37,5 \\
\hline $\begin{array}{l}\text { Estancia hospitalaria posquirúrgica } \\
\text { (días) }\end{array}$ & \multicolumn{2}{|c|}{$18,7 \pm 11,7$} \\
\hline Mortalidad a los 30 días posquirúrgicos & 0 & 0,0 \\
\hline
\end{tabular}

*ASA: American Society of Anesthesiologists
Tabla 3. Comparación por sexo de las características sociodemográficas, antecedentes personales y paraclínicos de los pacientes operados.

\begin{tabular}{|c|c|c|c|}
\hline Variable & $\begin{array}{c}\text { Femenino } \\
\qquad \begin{array}{c}\mathrm{N}=16 \\
\mathrm{n}(\%)\end{array}\end{array}$ & $\begin{array}{c}\text { Masculino } \\
\mathrm{N}=24 \\
\mathrm{n}(\%)\end{array}$ & Valor $p$ \\
\hline $\begin{array}{l}\text { Edad, en años } \\
\text { (Promedio y DE) }\end{array}$ & $68,8 \pm 12,9$ & $68,0 \pm 10,5$ & 0,8306 \\
\hline $\begin{array}{l}\mathrm{IMC}, \text { en } \mathrm{kg} / \mathrm{m}^{2} \\
\text { (Promedio y DE) }\end{array}$ & $23,4 \pm 2,8$ & $23,8 \pm 2,5$ & 0,6392 \\
\hline Bajo peso & $0(0,0)$ & $2(8,3)$ & 0,5076 \\
\hline Peso normal & $11(68,7)$ & $13(54,2)$ & 0,5121 \\
\hline Sobrepeso & $5(31,3)$ & $9(37,5)$ & 0,7462 \\
\hline Obesidad & $0(0,0)$ & $0(0,0)$ & -- \\
\hline \multicolumn{4}{|l|}{$\begin{array}{l}\text { Régimen de Seguridad } \\
\text { Social en Salud }\end{array}$} \\
\hline Contributivo & $10(62,5)$ & $12(50,0)$ & 0,5255 \\
\hline Subsidiado & $6(37,5)$ & $12(50,0)$ & \\
\hline Paciente remitido & $3(18,7)$ & $8(33,3)$ & 0,4732 \\
\hline Ingreso por urgencias & $13(81,2)$ & $18(75,0)$ & 0,7170 \\
\hline \multicolumn{4}{|l|}{$\begin{array}{l}\text { Antecedentes } \\
\text { personales }\end{array}$} \\
\hline Hipertensión arterial & $10(62,5)$ & $15(62,5)$ & 0,9999 \\
\hline Diabetes mellitus & $5(31,2)$ & $11(45,8)$ & 0,5121 \\
\hline Cardiovascular & $1(6,2)$ & $2(8,3)$ & 0,8087 \\
\hline Asma & $0(0,0)$ & $1(4,2)$ & 0,4142 \\
\hline EPOC & $1(6,3)$ & $0(0,0)$ & 0,4000 \\
\hline \multicolumn{4}{|l|}{$\begin{array}{l}\text { Hemoglobina }(n=33) \\
(g / L)\end{array}$} \\
\hline Prequirúrgica & $9,1 \pm 2,1$ & $9,5 \pm 1,4$ & 0,4738 \\
\hline Posquirúrgica & $10,2 \pm 0,8$ & $9,9 \pm 1,6$ & 0,4929 \\
\hline \multicolumn{4}{|l|}{$\begin{array}{l}\text { Albúmina } \\
\text { prequirúrgica (g/dl) }\end{array}$} \\
\hline$<2$ & $0(0,0)$ & 0,0 & -- \\
\hline $2-2,5$ & $10(62,5)$ & $1(4,2)$ & $<0,0001$ \\
\hline $2,6-3,0$ & $5(31,2)$ & $9(37,5)$ & 0,7462 \\
\hline $3,1-3,5$ & $7(43,7)$ & $7(29,2)$ & 0,5001 \\
\hline$>3,5$ & $0(0,0)$ & $2(8,3)$ & 0,5076 \\
\hline No reportado & $4(25,0)$ & $5(20,8)$ & 0,7601 \\
\hline
\end{tabular}

DE: desviación estándar; IMC: índice de masa corporal; EPOC: enfermedad pulmonar obstructiva crónica. 
Tabla 4. Comparación por sexo de la anestesia, la cirugía y la clasificación de la lesión en los pacientes operados.

\begin{tabular}{|c|c|c|c|}
\hline Variable & $\begin{array}{c}\text { Femenino } \\
N=16 \\
n(\%)\end{array}$ & $\begin{array}{c}\text { Masculino } \\
\mathrm{N}=24 \\
\mathrm{n}(\%)\end{array}$ & Valor $p$ \\
\hline \multicolumn{4}{|l|}{ Clasificación ASA } \\
\hline I & $6(37,5)$ & $8(33,3)$ & 0,4984 \\
\hline II & $5(31,2)$ & $5(20,8)$ & 0,4823 \\
\hline III & $3(18,7)$ & $7(29,2)$ & 0,7110 \\
\hline IV & $2(12,5)$ & $4(16,7)$ & 0,7210 \\
\hline Anestesia general & $16(100,0)$ & $23(95,8)$ & 0,4142 \\
\hline Gastrectomía total & $8(50,0)$ & $12(50,0)$ & 0,9999 \\
\hline \multicolumn{4}{|c|}{ Localización anatómica de la lesión } \\
\hline Unión esofagogástrica & $1(6,2)$ & $0(0,0)$ & 0,4000 \\
\hline Fondo gástrico & $5(31,2)$ & $7(29,2)$ & 0,8893 \\
\hline Cuerpo gástrico & $4(25,0)$ & $8(33,3)$ & 0,7294 \\
\hline Antro gástrico & $4(25,0)$ & $8(33,3)$ & 0,7294 \\
\hline No reportado & $2(12,5)$ & $1(4,2)$ & 0,5530 \\
\hline \multicolumn{4}{|c|}{ Clasificación de Borrmannn } \\
\hline Tipo 1 & $0(0,0)$ & $1(4,2)$ & 0,4142 \\
\hline Tipo 2 & $0(0,0)$ & $2(8,3)$ & 0,5076 \\
\hline Tipo 3 & $5(31,2)$ & $6(25,0)$ & 0,7275 \\
\hline Tipo 4 & $9(56,3)$ & $13(54,2)$ & 0,8980 \\
\hline No reportado & $2(12,5)$ & $2(8,3)$ & 0,6708 \\
\hline $\begin{array}{l}\text { Tipo histológico } \\
\text { Adenocarcinoma }\end{array}$ & $14(87,5)$ & $21(87,5)$ & 0,9998 \\
\hline \multicolumn{4}{|l|}{ Estadio } \\
\hline IIB & $6(37,5)$ & $10(41,6)$ & 0,7947 \\
\hline IIC & $0(0,0)$ & $1(4,2)$ & 0,4000 \\
\hline IIIA & $2(12,5)$ & $1(4,2)$ & 0,5530 \\
\hline IIIB & $6(37,5)$ & $9(37,5)$ & 0,9999 \\
\hline No reportado & $2(12,5)$ & $3(12,5)$ & 0,9999 \\
\hline Pérdida sanguínea & $2(12,5)$ & $0(0,0)$ & 0,1538 \\
\hline Complicaciones & $1(6,2)$ & $0(0,0)$ & 0,4000 \\
\hline Transfusiones & $9(56,3)$ & $13(54,2)$ & 0,8980 \\
\hline Prequirúrgica & $1(6,2)$ & $5(20,8)$ & 0,3725 \\
\hline Posquirúrgica & $8(50,0)$ & $8(33,3)$ & 0,3386 \\
\hline $\begin{array}{l}\text { Estancia hospitalaria } \\
\text { posquirúrgica (días) }\end{array}$ & $18,7 \pm 9,2$ & $18,7 \pm 13,4$ & 0,9998 \\
\hline $\begin{array}{l}\text { Mortalidad a los } 30 \\
\text { días posquirúrgicos }\end{array}$ & $0(0,0)$ & $0(0,0)$ & -- \\
\hline
\end{tabular}

El adenocarcinoma gástrico tiene una incidencia dos veces mayor en los hombres ${ }^{\mathrm{I}}$, una diferencia que ha sido explicada por el efecto de los estrógenos como factores protectores $\left(\mathrm{OR}=0,6 \mathrm{I}, \mathrm{IC}_{95} \% \mathrm{O}, 50-0,74\right)^{\mathrm{I}}{ }^{\mathrm{I}}$. En este estudio se observó mayor cantidad de hombres, con cáncer gástrico avanzado (6o \%), aunque con una tendencia descendente con respecto a los datos del periodo 2007-2009, donde se reportó un 72,I $\%{ }^{14}$.

Todos los participantes en los que se registró la histología presentaron adenocarcinoma, con estadio II con mayor frecuencia, similar a lo reportado por el centro de investigaciones oncológicas ${ }^{15}$, donde los participantes con adenocarcinoma gástrico presentaron con mayor frecuencia estadio III, seguido del II. Predominó la presentación proximal de la lesión, con un $62,5 \%$ en cardias, fondo y cuerpo, dato similar al reportado en el Hospital Universitario la Samaritana de Bogotá con un $56,4 \%{ }^{16}$, pero diferente de lo reportado en un estudio regional de Chile con 35,8 \% de localización corporal, 35,4\% de ubicación proximal (cardias o unión esofagogástrica) y 23,9\% antral ${ }^{17}$.

En el centro de investigaciones oncológicas ${ }^{15}$, el $63,3 \%$ de los pacientes requirieron gastrectomía total y el 36,7\% subtotal, mientras en nuestro estudio se presentó una distribución homogénea, requiriéndose de gastrectomía total y subtotal en un $50,0 \%$ cada una.

El cáncer gástrico avanzado, cuyo compromiso va más allá de la submucosa, es de mal pronóstico, con una tasa de sobrevida en Occidente a los cinco años de 5 a $20 \%$. Esto se relaciona con la escasa existencia de programas de detección precoz, debido a la inadecuada relación costo-beneficio en planes de tamizaje por su baja incidencia ${ }^{18}$. Por el carácter de este estudio, no se pudo evaluar la sobrevida en estos pacientes.

A nivel mundial se ha estudiado la relación de las comorbilidades con la displasia y el cáncer gástrico, y se ha encontrado que los niveles altos de glicemia [IOO-I25 $\mathrm{mg} / \mathrm{dl}$ ] representan un riesgo relativo del 2,3 para cáncer gástrico $\left[\mathrm{IC}_{95} \%\right.$ I,I47-4,457; $\mathrm{p}=0.019]^{19}$. Una de las teorías explicativas atribuye su relación con cáncer gástrico a una mayor ingesta de sal debido a la pérdida 
de la sensación de sabor, sin embargo, no existe evidencia confirmada ${ }^{20}$; por otro lado, el uso de medicamentos como la insulina, la metformina, las sulfonilureas, la aspirina y las estatinas, también puede influir en el riesgo de cáncer gástrico, pero la mayoría de ellos no se han estudiado exhaustivamente ${ }^{20}$. En nuestro estudio se encontró alta prevalencia de diabetes mellitus, tanto en hombres como en mujeres.

En general, independientemente del sexo, los pacientes ingresaron en buenas condiciones a su intervención quirúrgica, pues fueron clasificados en su mayoría como ASA I, con un promedio de hemoglobina prequirúrgica en 9,3 $\pm \mathrm{I}, 9 \mathrm{~g} / \mathrm{L}$ y albumina en rangos de 2,6-3,5 g/dL, lo que explicaría en parte el hecho de no reportar mortalidad en los primeros 30 días posteriores a la gastrectomía laparoscopia. Un estudio que evaluó la experiencia de 50 años en gastrectomía por adenocarcinoma gástrico mostró que los factores de riesgo para mortalidad intrahospitalaria fueron la hipoalbuminemia $(<3,5 \mathrm{~g} / \mathrm{dL})(\mathrm{OR} 2,4)$, la transfusión sanguínea intraoperatoria $(\mathrm{OR} 5,9)$ y

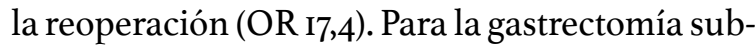
total distal los factores de riesgo para mortalidad intrahospitalaria fueron la hipoalbuminemia (OR 2,6), la transfusión sanguínea intraoperatoria (OR 2,46), la extirpación del bazo (OR 2,42) y la reoperación (OR 6,3) ${ }^{21}$. Cabe resaltar que en este estudio no se reportó reintervención ni requerimiento de transfusión intraoperatoria.

Llama la atención la estancia postquirúrgica de I8,7 \pm II,7 días, en comparación a un estudio realizado en Chile en donde fue de $7,3 \pm 2,8$ días ${ }^{22}$, pero más acorde a la estancia hospitalaria postoperatoria reportada en Perú, con un promedio de II,7 $\pm 6,3$ días (rango 5-37) días para la gastrectomía distal y I4,8 \pm II,3 días (rango 7-56 días) para la gastrectomía total ${ }^{23}$.

Como limitante del estudio, es importante recalcar el no diligenciamiento de la historia clínica, reportado en un 6,8\%. La función de la historia clínica es ser fuente de información para identificar al paciente, justificar el diagnóstico y tratamiento, además de documentar los resultados de la asistencia médica. No se encontraron datos en la literatura sobre la evaluación del diligenciamiento adecuado de la historia clínica en los departamentos quirúrgicos de los Hospitales Universitarios Colombianos, sin embargo, los estándares de calidad tienen como referencia el Ioo \% de diligenciamiento adecuado de la historia clínica.

\section{Cumplimiento de normas éticas}

Consentimiento informado: Este estudio es una revisión retrospectiva de historias clínicas, por lo que no implica un riesgo para los pacientes y no se requiere consentimiento informado.

Declaración de conflicto de intereses: Los autores declaran que no tienen conflictos de intereses.

Fuentes de financiación: Recursos propios de los autores.

\section{Referencias}

I. Ferlay J, Soerjomataram I, Dikshit R, Eser S, Mathers $\mathrm{C}$, Rebelo M, et al. Cancer incidence and mortality worldwide: Sources, methods and major patterns in GLOBOCAN 2012. Int J Cancer. 2015;136:E359-86.

http://doi.wiley.com/I0.I002/ijc.292IO

2. Bray F, Piñeros M. Cancer patterns, trends and projections in Latin America and the Caribbean: a global context. Salud Publica Mex. 2016;58:104-17.

http://dx.doi.org/Io.2II49/spm.v58i2.7779

3. Instituto Nacional de Cancerología ESE. Análisis de la situación del cáncer en Colombia 2015. Primera edición. Bogotá, D.C.: Instituto Nacional de Cancerología ESE; 2017. Disponible en: https://www.cancer.gov.co/ Situacion_del_Cancer_en_Colombia_2015.pdf

4. Oliveros R, Navarrera LF. Diagnóstico, estadificación y tratamiento del cáncer gástrico en Colombia desde 2004 a 2008 (REGATE-Colombia). Rev Colomb Gastroenterol. 20I2;27:269-74.

5. Pardo C, Cendales R. Incidencia, mortalidad y prevalencia de cáncer en Colombia 2007-20II. Vol. I, Instituto Nacional De Cancerologia. 2015. I48 p. Disponible en: https://www.cancer.gov.co/files/libros/archivos/incidenciai.pdf

6. Torres W. Perfil epidemiologico del Distrito de Cartagena - año 20I4. Disponible en: https://www. dadiscartagena.gov.co/images/docs/saludpublica/perfil_epidemiologico_20I4.pdf

7. Ramos K. Cancer gastrico: caracteristicas clinicoepidemiologicas e histopatologicas en el Hospital Universitario del Caribe desde enero de 2007 a diciembre de 2009. Universidad de Cartagena; 20Io. Disponible en: https://repositorio.unicartagena.edu.co/bitstream/ handle/II227/II67/TRABAJO\%2OKATYA\%20RAMOS. pdf? sequence $=\mathrm{I} \&$ isAllowed $=\mathrm{y}$ 
8. Quan Y, Huang A, Ye M, Xu M, Zhuang B, Zhang P, et al. Comparison of laparoscopic versus open gastrectomy for advanced gastric cancer: an updated meta-analysis. Gastric Cancer. 2016;19:939-50.

https://doi.org/I0.IO07/sioI20-0I5-05I6-x

9. Özer İ, Bostancı EB, Ulaş M, Özoğul Y, Akoğlu M. Changing trends in gastric cancer surgery. Balkan Med J. 20I7;34:10-20.

https://doi.org/I0.4274/balkanmedj.20I5.I46I

Io. Thriff A, El-Seraq H. Burden of gastric cancer. Clin gastroenterol hepatol. 2020;18:534-42.

https://doi.org/IO.IoI6/j.cgh.2019.07.045

II. Adrada J, Calambas F, Diaz J, Delgado D, Sierra C. Caracteristicas sociodemograficas y clinicas en una poblacion con cancer gastrico en el Cauca, Colombia. Rev Col Gastr. 2008;23:309-I4

I2. Duell EJ, Bonet C, Muñoz X, Lujan-Barroso L, Weiderpass $\mathrm{E}$, Boutron-Ruault MC, et al. Variation at ABO histo-blood group and FUT loci and diffuse and intestinal gastric cancer risk in a European population. Int J Cancer. 2015;136:880-93. https://doi.org/IO.IO02/ijc.29034

I3. Brusselaers N, Maret-Ouda J, Konings P, El-Serag HB, Lagergren J. Menopausal hormone therapy and the risk of esophageal and gastric cancer. Int J Cancer. 20I7;I40:I693-9. https://doi.org/IO.IOO2/ijc.30588

I4. Galeano-Espitia N. Relacion entre la afectacion del omento, estadio y las caracteristicas clinicas e histopatologicas del cancer gastrico avanzado. 20I7. Disponible en: https://repositorio.unicartagena.edu.co/bitstream/ handle/II227/59I7/TRABAJO\%2OFINAL\%2ONOHEMI\%20GALEANO\%2oESPITIA.pdf?sequence=I

I5. Carrillo G, Bayona H, Arias E. Perfil y carga de la enfermedad de personas con cáncer gástrico sometidas a gastrectomía. Rev Fac Med. 2018;66:13-8. https://doi.org/I0.I5446/revfacmed.v66nI.60273
I6. Martínez-Marín JD, Garzón-Olarte MA, Lizarazo-Rodríguez JI, Marulanda-Gómez JC, Molano-Villa JC, Rey-Tovar MH, et al. Características de los pacientes con cáncer gástrico del departamento de Cundinamarca, remitidos al Hospital Universitario de la Samaritana entre los años 2004 y 2009. Rev Col Gastroenterol. 20IO;25:344-8.

I7. Muller B. et al. Registro de evaluación de tratamiento de cáncer gástrico en Chile: Características basales de 523 pacientes. Rev Chil Cir. 20II;63:I47-53. http://dx.doi.org/I0.4067/So718-402620II000200004

I8. Otero W. Cáncer gástrico en Colombia: un diagnóstico tardío que amerita el compromiso del estado. Rev Col Gastroenterol. 2008;23:302-4.

19. Yang P, Zhou Y, Chen B, Wan HW, Jia GQ, Bai HL, et al. Overweight, obesity and gastric cancer risk: Results from a meta-analysis of cohort studies. Eur J Cancer. 2009;45:2867-73. http://dx.doi.org/Io.IoI6/j.ejca.2009.04.0I9

20. Tseng CH, Tseng FH. Diabetes and gastric cancer: The potential links. World J Gastroenterol. 20I4;20:I7OI-II. https://doi.org/IO.3748/wjg.v20.i7.I7OI

2I. Ruiz E, Payet C, Montalbetti JA, Celis J, Payet E, Berrospi $\mathrm{F}$, et al. Morbilidad post operatoria y mortalidad intrahospitalaria de la gastrectomía por adenocarcinoma gástrico: Análisis de 50 años. Rev Gastroenterol del Perú. 2004;24:197-2IO.

22. Escalona A, Baez S, Pimentel F, Calvo A, Boza C, Viñuela E, et al. Laparoscopic gastrectomy for gastric cancer. Rev Chil Cir. 2008;60:188-93. http://dx.doi.org/I0.4067/So718-40262008000300004

23. Portanova M, Vargas F, Lombardi E, Carbajal R, Palacios N, Rodriguez C, et al. Tratamiento quirúrgico del cáncer gástrico en un servicio especializado: experiencia del Hospital Rebagliati. Rev gastroenterol Perú. 2005;25:239-297 